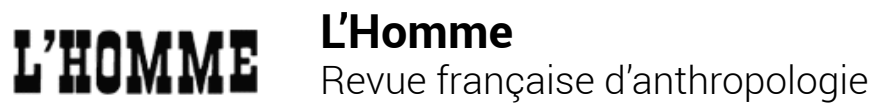

175-176 | juillet-septembre 2005

Vérités de la fiction

\section{Nigel Rapport, ed., British Subjects: An Anthropology of Britain}

Oxford-New York, Berg, 2002, XII + 340 pages

Josiane Massard-Vincent

\section{CpenEdition}

\section{Journals}

Édition électronique

URL : http://journals.openedition.org//homme/2078

DOI : 10.4000//homme.2078

ISSN : 1953-8103

Éditeur

Éditions de l'EHESS

Édition imprimée

Date de publication : 15 octobre 2005

Pagination : 557-560

ISBN : 2-7132-2035-1

ISSN : 0439-4216

Référence électronique

Josiane Massard-Vincent, « Nigel Rapport, ed., British Subjects: An Anthropology of Britain », L'Homme

[En ligne], 175-176 | juillet-septembre 2005, mis en ligne le 30 novembre 2006, consulté le 24

septembre 2020. URL : http://journals.openedition.org//homme/2078 ; DOI : https://doi.org/10.4000/ Ihomme.2078

Ce document a été généré automatiquement le 24 septembre 2020.

(c) École des hautes études en sciences sociales 


\title{
Nigel Rapport, ed., British Subjects: An Anthropology of Britain
}

\author{
Oxford-New York, Berg, 2002, XII + 340 pages
}

Josiane Massard-Vincent

\section{RÉFÉRENCE}

Nigel Rapport, ed., British Subjects: An Anthropology of Britain, Oxford-New York, Berg, 2002 , XII + 340 p., index, ill.

1 RÉUNISSANT quinze contributions à l'ethnologie de la Grande-Bretagne - sans l'Irlande du Nord -, cet ouvrage est dû à Nigel Rapport, dont le riche parcours scientifique atteste d'une réflexion exigeante, originale et souvent stimulante. Les travaux rassemblés confirment dans une grande mesure le renouveau de l'anthropologie britannique apparu à l'université de Manchester à la fin des années 1970, à l'instigation notamment d'Anthony P. Cohen - dédicataire du volume -, épaulé par Nigel Rapport lui-même depuis le début des années 1990. Soucieux de réévaluer à la fois les déterminismes globalisants - tels la classe, le religieux, les moyens de production, etc. - et la démarche monographique, Anthony Cohen a prôné une observation attentive de la localité ou de milieux circonscrits. Les études de cas analysées ici vont dans ce sens bien que l'ethnographie y soit parfois restituée de manière parcimonieuse, ou très condensée, laissant une large place à de longs développements théoriques et/ou épistémologiques. Elles ont été réparties selon cinq entrées thématiques, proposées initialement par l'éditeur scientifique: "Nationalisme, contestation et mise en acte de la tradition ", "Stratégies de modernité: héritage, loisir, dissociation », "L'appropriation du discours ", "Méthodologies et ethnométhodologies", «La fabrication et la déconstruction de la communauté : ethnicité, religiosité, localité ».

2 La dimension nationale de l'entreprise est annoncée dès le titre avec un goût de la polysémie : "Sujets britanniques » n'est-il pas à triple entente de thèmes de recherche, de ressortissants du royaume, mais aussi de sujets en tant qu'acteurs, par opposition à 
« objets », sachant que la plupart des participants à ce volume entrent, on le verra, dans la deuxième catégorie? Nigel Rapport confirme son parti pris en intitulant son introduction "Best of British!", jouant là sur le double sens d'une expression vernaculaire : «Une sélection britannique » et « On vous souhaite bien du plaisir ! ». Le ton est donné et la suite est à l'avenant, ainsi des motifs de l'entreprise énoncés dans le "cahier des charges" soumis aux auteurs: "l'anthropologie en/de et sur la GrandeBretagne est centrale dans les préoccupations de la discipline. [Elle] pourrait inclure tout ce qui concerne l'anthropologie comme discipline [...]. Réalisée par ceux dont la langue maternelle est l'anglais (ou le gallois, ou le hindi suivant le cas) ou par d'authentiques bilingues, [elle] fixe des critères d'excellence en termes d'ethnographie subtile et d'analyse fine que d'autres pourraient chercher à égaler. Loin d'être une anomalie, une anthropologie "chez soi" en Grande-Bretagne pourrait être considérée comme paradigmatique » (p. 6).

3 Il s'agit de montrer que la Grande-Bretagne recèle tous les thèmes d'étude dignes d'intérêt : « rien de ce qui fait l'expérience humaine ne peut [en] être absent » (p. 8). Le lecteur est alors en droit d'être perplexe, mais la phrase suivante dissipe l'ambiguïté : "J'entends par là », poursuit Nigel Rapport, «que ce qui est central à la découverte anthropologique est la psyché humaine et cela est universel » (ibid.). Reprenant à son compte le propos de Marc Augé selon lequel « ce sont les individus qui restent le ciment anthropologique", Nigel Rapport défend une "anthropologie existentielle» de préférence à une "anthropologie culturelle ", rejetant à son tour toute interprétation des rapports sociaux par le recours à des superstructures (p. 10), et poursuivant avec les implications de ce point de vue : "les gens sont tous et toujours les anthropologues de leur vie "-cf. l'un des sens de subject dans le titre - "toujours engagés dans l'ethnométhodologie» (p. 7). Il existe certes des pratiques tels le bed \& breakfast ou le rugby, mais elles ne sont pas déterminantes, n'étant que des " phénomènes de surface " et des «accidents de l'histoire» (p.8) eu égard à la nécessité de rendre compte des « expériences significatives des individus » (p.9). De ce plaidoyer pour une «science du particulier », il s'ensuit que la "Grande-Bretagne, - ce que c'est, qui en fait partie et comment - ne saurait être essentialisée ou absolument définie, il n'y a pas de GrandeBretagne représentative d'un point de vue anthropologique » et on ne doit considérer ni la Grande-Bretagne ni "britannique » comme donnés. Avec Clifford Geertz, Nigel Rapport rappelle que « le lieu de l'étude n'est pas l'objet de l'étude » (p.17). En d'autres termes, et en dépit du titre, que le lecteur ne s'attende pas à trouver là une contribution à la « britannologie »" !

4 Cette position de principe n'oriente toutefois pas le contenu des articles proprement dits dans le sens où, exception faite justement de celui de Nigel Rapport lui-même ("The Body of the Village Community», pp. 299-319), tous sont centrés sur des groupes, des usages sociaux ou des institutions localisés : comment prétendre que leur cadrage territorial, linguistique, politique et historique en Grande-Bretagne est extérieur aux problématiques développées ? La confrontation de cette hypothèse à trois des contributions - la première sur les admirateurs de la monarchie (Anne Rowbottom, pp. 31-47), une autre sur le sentiment national dans l'île de Man (Susan Lewis, pp. 49-65), une encore sur l'essence du mouvement quaker (Peter Collins, pp. 281-298) montre que des spécificités empiriques ancrées dans une histoire et un contexte «nationaux » font partie intégrante non seulement de l'enquête mais de la réflexion. Sans étayer une définition "substantialiste » de ce que pourrait être une identité 
britannique, l'analyse de ce que Nigel Rapport considère comme des "épiphénomènes " (p. 8) ressortit davantage à l'anthropologie sociale qu'à une démarche « existentielle ».

5 Envisageons maintenant l'argument de la supériorité que confère la parfaite maîtrise de la langue au chercheur autochtone observant des faits locaux. L'immersion dans le quotidien familier peut de fait rendre «naturel » le recours à des concepts indigènes dont certains avaient pourtant été judicieusement «déconstruits» par d'autres anthropologues britanniques. Ainsi le concept de "classe ", omniprésent dans le discours spontané, et dont Marylin Strathern a montré, à partir de ses enquêtes à Elmdon (Sussex), qu'il relevait du sens commun ${ }^{2}$. La charge idéologique dont il est investi par les sujets/informateurs en rend la validité heuristique problématique pour l'observateur/anthropologue. Deux des auteurs du volume reprennent néanmoins au premier degré des éléments de ce lexique : Anne Rowbotton quand elle écrit « upperworking class » et « lower-working class background » (p. 34) et Helene Wulff, « upperworking class and middle-middle class » (p. 70). De la même manière, Nigel Rapport ("art cit.») revient au vocable de " communauté », dont le maniement comme outil d'analyse ne va pas de soi non plus ${ }^{3}$. Il est aussi d'autres notions employées par les auteurs, tels public ou national, qui malgré leur ubiquité dans le discours savant ou ordinaire et leur apparente limpidité pour des locuteurs d'autres langues européennes, n'ont pas le même sens d'un côté ou l'autre de la Manche. Pour un lecteur non anglophone, l'utilisation de ces concepts émiques est source d'ambiguïté.

6 Si Nigel Rapport et les auteurs du volume n'ont, en général, pas jugé le détour par la contextualisation nécessaire, c'est sans doute parce qu'ils sous-estiment ce qui est au mieux la méconnaissance mais le plus souvent l'ignorance de l'ethnographie anglaise ou britannique par leurs homologues étrangers. Ceux-ci n'ont pas toujours suivi les travaux de représentants d'autres disciplines (géographie rurale et sociologie) et d'antropologues réalisés en Grande-Bretagne à partir d'études de terrain depuis la Seconde Guerre mondiale. Paradoxalement, le malentendu tient peut-être aussi au double statut de la langue anglaise : nombre de chercheurs non anglophones manient un sabir basé sur l'anglais mais ignorent l'anglais « réel » dans ses rapports étroits avec une culture - ce qu'est un idiome de terrain appris conjointement avec l'observation de la société qui le pratique. Or c'est bien cette autre langue, vernaculaire, que les auteurs de l'ouvrage parlent à certains moments au risque de rester entre soi : que recouvrent pour un lecteur non britannique des institutions indigènes tels le Working Men's Club ou le Derby \& Joan Club (Dawson, p. 118) ? À l'encontre du propos de Nigel Rapport, le nondit partagé par les auteurs tendrait peut-être à prouver la difficulté à s'affranchir dans l'écriture, sinon d'une identité, au moins d'une culture commune: celle-ci est sousjacente mais non explicitée dans aucun des textes. L'ignorer équivaudrait à nier la part d'intersubjectivité qui préside à la relation chercheur/informateur lors du recueil de données.

7 Malgré ces questions, la promesse de l'introduction Best of British est largement tenue : l'ouvrage devrait faire date dans l'histoire de la discipline. Il constitue un apport considérable par la diversité des échelles et des approches, par la finesse et la richesse des observations qu'il restitue, par les problématiques qu'il développe et par l'esprit critique dont il fait preuve, par exemple eu égard à la partialité de certains anthropologues dans l'étude des minorités. En dépit des certitudes de Nigel Rapport quant à l'inexistence d'une identité britannique du point de vue de la discipline, certaines pratiques sociales, peut-être spécifiquement britanniques, méritent d'être 
évoquées, même de manière concise, voire allusive, comme c'est parfois le cas ici. Outre des titres déjà cités, mentionnons la quête d'une esthétique "nationale » dans le Royal Ballet (Helena Wulff, pp. 67-83), ou encore le rapport entre ethnographie et roman tel que l'incarne le personnage du vicaire (Nigel Rapport, « art cit. »). D'autres sujets, bien qu'également intégrés dans l'univers social britannique, renforcent en même temps des intérêts classiques de la discipline : ainsi de l'assignation de certains "affects " et valeurs à des groupes « ethniques » - en l'occurence gallois - analysée par Carol Trosset et Douglas Caulkins (pp. 239-256) ou de la manipulation et de la fluidité de l'identité ethnique en diaspora (exemple des Arméniens) par Vered Amit (pp. 263-279). D'autres thèmes renvoient à des problématiques contemporaines plus larges tels la muséification d'objets quotidiens comme attributs d'une identité (Sharon Macdonald, pp. 89-106) ou les processus d'appropriation par le savoir populaire d'innovations scientifiques (Jeannette Edwards, pp. 163-179). Autre sujet transversal ayant également un écho européen, quand Christine Brown (pp.223-238) explore les questions déontologiques soulevées par un projet d'enquête en milieu psychiatrique "fermé ». Quant à la contribution de Jenny Hockey à partir d'une expérience d'observation (vraiment) participante dans une maison de retraite (pp. 209-222), elle interroge les rapports entre entretien ethnographique et immersion.

Anthony P. Cohen plaidait avec raison pour la prise en compte par ses collègues britanniques de l'hétérogénéité et de la complexité de leur propre société, y compris dans ses composantes ordinaires, territorialisées ou transversales, et non plus seulement dans ses confins géographiques ou ethniques. L'ouvrage dirigé par Nigel Rapport prouve qu'il a été entendu, au moins dans le choix des objets: il offre une contribution remarquable à la connaissance d'un univers géographiquement voisin mais socialement inconnu. En termes disciplinaires, il participe des interrogations théoriques et épistémologiques qui entraînent l'anthropologie hors des sentiers battus. En comblant une partie du hiatus entre langue anglaise et culture britannique, il peut aussi participer au remplacement progressif d'un idiome à la fois familier et opaque, l'anglais «international», par une langue "vivante». Compte tenu de la démarche initiale, quelle image de l'anthropologie d'Outre-Manche cet ouvrage façonne-t-il ? En prônant l'universalisme des relations interpersonnelles, Nigel Rapport a peut-être réussi à injecter un particularisme culturel dans ses "sujets ", à entendre dans tous les sens du terme.

\section{NOTES}

1. . Cf. Anthony P. Cohen, "Travaux récents en anthropologie rurale de la Grande-Bretagne », in M. Bodiguel \& Ph. Lowe, eds, Campagne française, campagne britannique : histoires, images, usage au crible des sciences sociales, Paris, L'Harmattan, 1989 : 293-305.

2. . Cf. Marylin Strathern, "The Place of Kinship : Kin, Class and Village Status in Elmdon, Essex ", in A. P. Cohen, ed., Belonging, Identity and Social Organisation in British Rural Cultures, Manchester, Manchester University Press, 1982 : 270-274. 
3. . Cf. aussi Marylin Strathern, "Localism Displaced: A "Vanishing Village" in Rural England", Ethnos, 1984, 1-2: 43-61.

4. .Cf. Anthony P. Cohen, "La tradition britannique et la question de l'autre ", in Martine Segalen, ed., L'Autre et le Semblable : regards sur l'ethnologie des sociétés contemporaines, Paris, CNRS Éd.: 39 . 